\title{
Faecopneumothorax revealing a neglected traumatic diaphragmatic hernia
}

\begin{abstract}
Introduction: Tension fecopneumothoraxis a rare but serious complication of traumatic diaphragmatic hernias. The diagnosis of the hernia can be delayed from a few days to some years and will be made on occasion of complications representing the dramatic evolution of the "latent stage" of disease. Only few cases of post-traumatic faecopneumothorax are described in the literature. This clinical evolution is associated to a significant increase in morbidity ( $30-80 \%$ of cases). This is particularly the case with our patient.
\end{abstract}

Case presentation: We report the case of a 26-year-old who had a trauma of the left hypochondrium about a year ago and who presented an acute intestinal occlusion, an $\mathrm{X}$ ray abdomen and chest was performed, showing abundant hydropneumothorax, thethoracoabdominal scan reveals an abundant effusion with heterogeneous density in the left pleural cavity, associated with an intrapleural hernia of the large intestine

Discussion: Tension fecopneumothorax is a very rare complication of traumatic diaphragmatic hernia, only few cases are described in the literature, the mechanisms of the injury can blunt or penetrating, it occurs after intrapleural perforation of a strangulated colon, and very often its life threatening.

Conclusion: According to our knowledge and review of the literature, Tension fecopneumothorax complicating a traumatic diaphragmatic hernia is very rare; the diagnosis is made by thoracic radiography and thoracoabdominalCT, which also allow orientation of the therapeutic attitude with non-negligible post-operative complications.

\section{Introduction}

Diaphragmatic rupture, is relatively rare, and can represent a lifethreatening situation. The first diaphragmatic injury with abdominal organ herniation was described by Sennertus. ${ }^{1}$ Early diagnosis is often difficult; it may be delayed for a few days to years and only be made following a complication. The most serious complication of a diaphragmatic hernia is strangulation, and the occurrence of symptoms of intestinal obstruction or pulmonary compression. This case outlines the necessity to early diagnose the traumatic diaphragmatic hernia to avoid complications

\section{Case report}

The 28-year-old male presented to the emergency with complaints of dyspnea at rest, pain in the left upper abdomen and vomiting. His past medical history was free except that he was attacked by a knife in the left hypochondrium about a year ago causing a superficial injury sutured at the emergency. He denied any systemic diseases, recent history of surgical procedures.

First a chest X-ray revealed a left hydropneumothorax and thoracic drainage was performed and bring heterogeneous matter and the patient begin to install an acute intestinal occlusion.

Then the patient was shifted for a computed tomography (CT) of thorax and abdomen (Figure1) which showed herniation in the left lower lung zone of the bowel loops through the disrupted left hemi diaphragm, with multiple air fluid levels. The herniated abdominal contents are positioned against the posterior wall of the thorax (dependent viscera sign).The collar at the base of the herniated bowel is more difficult to detect on the axial CT image than on reformatted images in other planes. Default of enhancement of the wall signs the ischemia. Diagnosis of complicated diaphragmatic hernia was
Volume 6 Issue 2 - 2019

\author{
B Chaoui,' I Nassar, ${ }^{2}$ N MoatassimBillah ${ }^{2}$ \\ 'Departement of radiology, Military training hospital \\ Mohamed-V, UMV, Rabat, Morocco \\ ${ }^{2}$ Department of central Radiology, CHU IbnSina , UMV Rabat, \\ Morocco
}

Correspondence: B Chaoui, Department of radiology, Military
training hospital Mohamed-V, UMV, Rabat, Morocco, Email chaouibadr@hotmail.com

Received: November 21, 2018 | Published: March 26, 2019 made. Emergency surgery was decided with reduction of the hernia, Segmental colic resection. The patient was extubated and hospitalized. On follow-up, vital signs were stable and radiological examination was normal (Figure 2). The patient was discharged on the third day postoperatively.

\section{Discussion}

Diaphragmatic rupture with abdominal organ herniation was first described by Sennertus in $1541,2,3$ Post traumatic diaphragmatic hernia results from blunt trauma injuries in $80 \%$ cases. Penetrating injuries such as stab wounds are associated with rest $20 \%$ cases. ${ }^{4}$ They mainly sit in the left side (In 70 to $90 \%$ of cases) due to the protective role of the liver on the right and stomach, colon, omentum are the most involved. ${ }^{5}$

The diagnosis can be delayed and revealed by a complication as it was precisely the case with our patient. The delayed diagnosis explained by Grimes in $1974,{ }^{6}$ who described 3 phases of the diaphragmatic rupture. The acute phase is the time of the injury. The delayed phase is associated with transient herniation of the viscera thus accounting for absence or intermittent nonspecific symptoms (shortness of breath, chest or abdominal pain, nausea, vomiting, and fever). The obstruction phase signifies complication of a long standing herniation, manifesting as obstruction, strangulation and rupture. ${ }^{3}$ the foecopneumothorax is a rare complication, and it can be lifethreatening. In our patient first we performed a radiography showing a hydropneumothorax and differential diagnosis was set with tumoral origin (young patient with no toxic history), infectious origin such as tuberculosis (no history of tuberculous contagion), no inflammatory disease or recent trauma. The boerhaavesyndrom or spontaneous rupture of the esophagus that go along with pneumo-mediastinum. $\mathrm{CT}$ is the exam of choice can shows indirect signs, the dependent 
viscera sign, $100 \%$ sensitive, ${ }^{7}$ and the herniated viscera lies against the posterior thoracic wall in a dependent position, as it is no longer supported by the diaphragm. The collar sign, $67 \%$ sensitive, ${ }^{7}$ is collarlike appearance of herniated organs at the level of the diaphragm.
And the direct sign is the visualization of the diaphragmatic defect. These signs are also hardly to detect on the axial CT image than on reformatted images in other planes on MPRs.
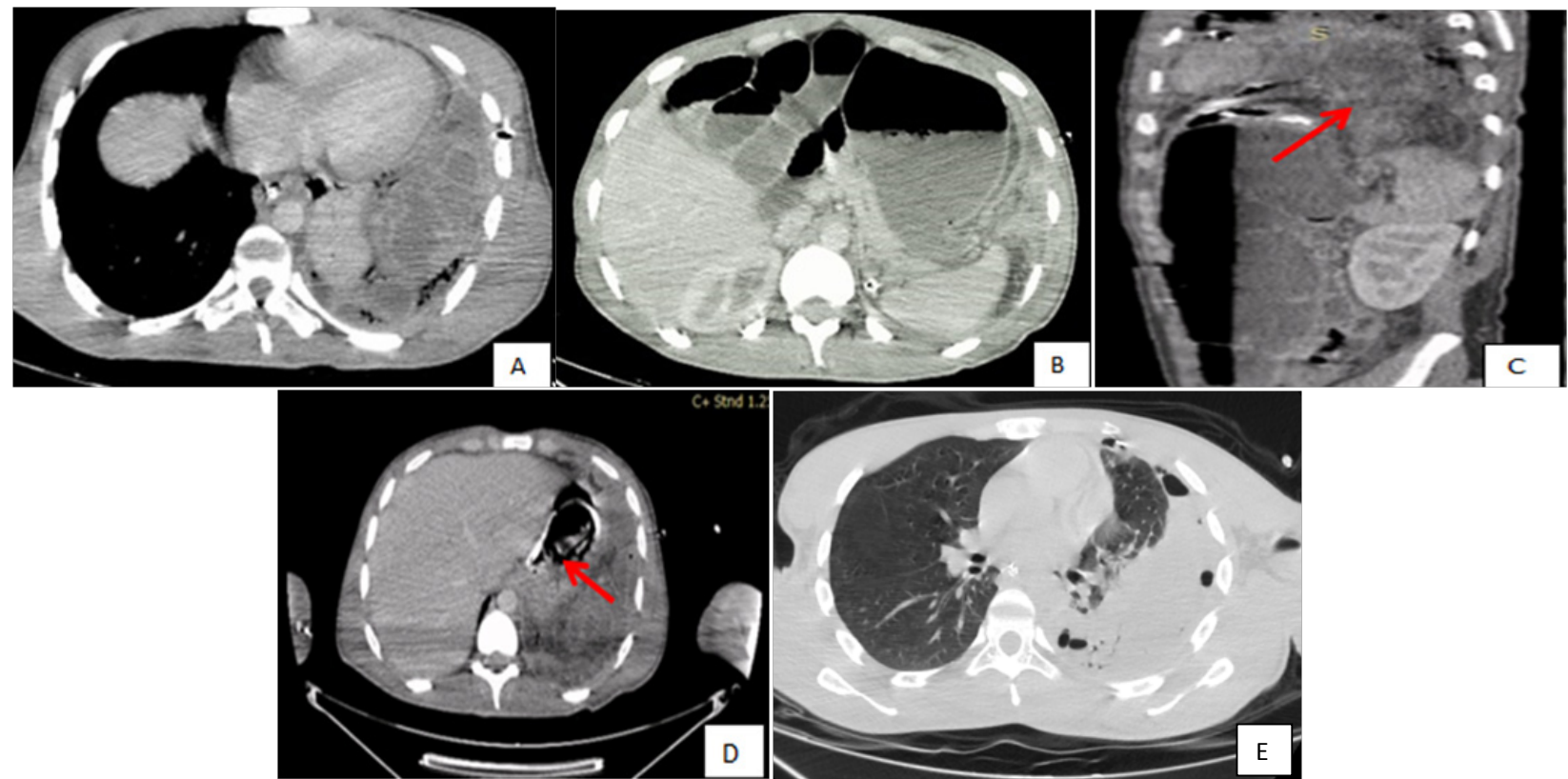

Figure I A.B(axial); $C($ Sag):CT scan shows a left hydropneumothorax, and collapse of the left lung, contralateral shift of the mediastinal contents under tension and left anterior diaphragmatic rupture (B.arrow)with herniated perforated colon(C.arrow), D: (arrow)The nasogastric tube lying just below the cardia (stomach is not involved in the hernia) $\mathrm{E}$ : $\mathrm{CT}$ in a lung window shows air next to herniated perforated colon.
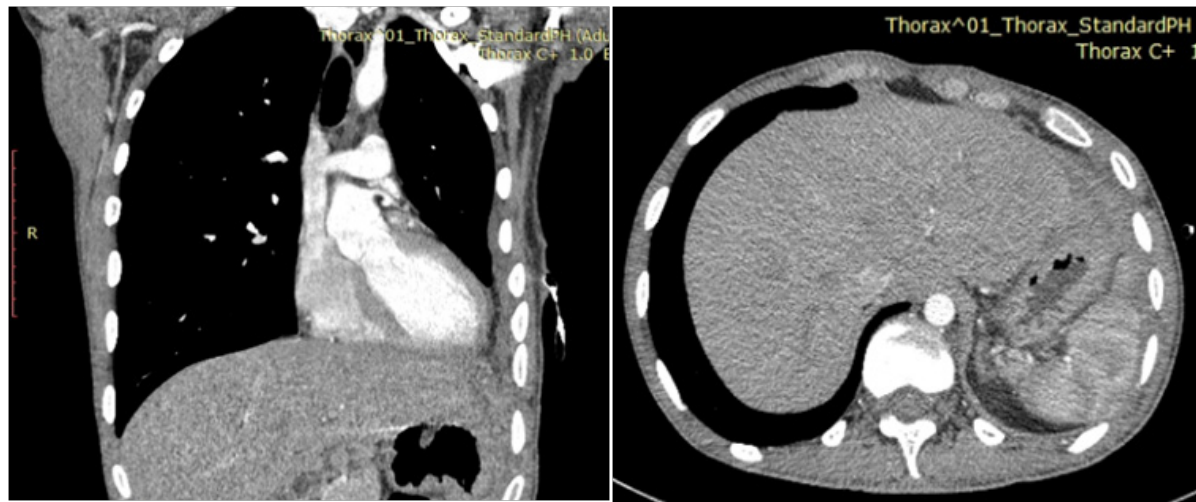

Figure 2 Postoperative CT scan showing expansion of the previously collapsed left lung.

\section{Conclusion}

Tension faecopneumothorax is a rare but serious complication following post-traumatic diaphragmatic hernia from a penetrating stab wound to the chest. This article highlights the point to always have in mind in front of chest pain, occlusive syndrome, and history of trauma, to think of neglected post traumatic hernia complicated with a faecopneumothorax.

\section{Acknowledgments}

None.

\section{Conflicts of interest}

The author declares that there is no conflicts of interest regarding the publication of this paper.

\section{References}

1. Desser TS, Edwards B, Hunt $\mathrm{S}$, et al. The dangling diaphragm sign: sensitivity and comparison with existing CT signs of blunt traumatic diaphragmatic rupture. Emerg Radiol. 2010;17(1):37-44.

2. Desir, Amandine, Ghaye Benoit. CT of Blunt Diaphragmatic Rupture. Radiographics. 2012;32(2):477-498.

3. Christie DB, Chapman J, Wynne JL, et al. Delayed right-sided diaphragmatic rupture and chronic herniation of unusual abdominal contents. J Am Coll Surg. 2007;204(1):176.

4. Goh BK, Wong AS, Tay KH, et al. Delayed presentation of a patient with a ruptured diaphragm complicated by gastric incarceration and perforation after apparently minor blunt trauma. CJEM. 2004;6(4):277-280.

5. Jeyarajah R, Harford WV. Abdominal hernias and gastric volvulus. In: Sliesenger and Fordtran's gastrointestinal and liver disease. 8th ed. Saunders, Elsevier; 2006. p. 477-481.

6. Nursal TZ, Ugurlu M, Kologlu M, et al. Traumatic diaphragmatic hernias: a report of 26 cases. Hernia. 2001;5(1):25-29. 